\title{
The effect of nasal tramazoline with dexamethasone in obstructive sleep apnoea patients
}

\author{
loannis Koutsourelakis, Aliki Minaritzoglou, Georgios Zakynthinos, \\ Emmanouil Vagiakis and Spyros Zakynthinos
}

Affiliation: Center of Sleep Disorders, Medical School of Athens University, Dept of Critical Care and Pulmonary Services, Evangelismos Hospital, Athens, Greece.

Correspondence: I. Koutsourelakis, Medical School of Athens University, Center of Sleep Disorders, Evangelismos Hospital, 45-47 Ipsilandou Str, GR 106 75, Athens, Greece. E-mail: ykoutsourelakisdyahoo.gr

ABSTRACT Although there is a strong correlation between oral/oro-nasal breathing and apnoea/ hypopnoea index in patients with obstructive sleep apnoea and normal nasal resistance at wakefulness, it remains unknown whether the pharmacological prevention of potential nasal obstruction during sleep could decrease oral/oro-nasal breathing and increase nasal breathing and subsequently decrease the apnoea/ hypopnoea index. This study evaluated the effect of a combination of a nasal decongestant with corticosteroid on breathing route pattern and apnoea/hypopnoea index.

21 patients with obstructive sleep apnoea (mean apnoea/hypopnoea index 31.1 events per hour) and normal nasal resistance at wakefulness were enrolled in a randomised crossover trial of 1 weeks' treatment with nasal tramazoline and dexamethasone compared with 1 weeks' treatment with nasal placebo. At the start and end of each treatment period, patients underwent nasal resistance measurement and overnight polysomnography with attendant measurement of breathing route pattern.

Nasal tramazoline with dexamethasone was associated with decrease in oral/oro-nasal breathing epochs and concomitant increase in nasal breathing epochs, and mean decrease of apnoea/hypopnoea index by $21 \%$. The change in nasal breathing epochs was inversely related to the change in apnoea/hypopnoea index $\left(\mathrm{R}_{\mathrm{s}}=0.78 ; \mathrm{p}<0.001\right)$.

In conclusion, nasal tramazoline with dexamethasone in OSA patients with normal nasal resistance at wakefulness can restore the preponderance of nasal breathing epochs and modestly improve apnoea/ hypopnoea index.

@ERSpublications

Nasal tramazoline with dexamethasone in OSA patients can restore nasal breathing epochs and improve AHI modestly http://ow.ly/mxu4T

Received: Sept 082012 | Accepted after revision: Jan 272013 | First published online: Feb 082013

Clinical trial: The present study is registered on the publicly accessible database www.clinicaltrials.gov (NCT01601509).

Conflict of interest: None declared.

Copyright @ERS 2013 


\section{Introduction}

Humans preferentially breathe via the nasal route, the purpose being to filter, warm and humidify the inspired air [1]. During sleep, healthy subjects free of nasal disease are estimated to inhale via the mouth only $\sim 4 \%$ of the total ventilation [2], but, when nasal obstruction occurs, the proportion of oral breathing increases and snoring with obstructive apnoeas appear [3,4]. Thus, increased nasal resistance is considered an independent risk factor for obstructive sleep apnoea (OSA) [5].

Despite the relationship between nasal obstruction and OSA, the therapeutic effect of improving nasal airway patency on OSA severity remains a point of conjecture [6]. In fact, in OSA patients with rhinitis administration of intranasal corticosteroids has been shown to improve sleepiness and reduce the apnoea/ hypopnoea index (AHI) [7], whereas the use of topical decongestants, external nasal dilators and nasal surgery has provided inconsistent results [8].

The present authors have demonstrated a strong correlation between AHI and oral/oro-nasal breathing epochs in patients with OSA and normal nasal resistance at wakefulness [9]. Additionally, KOHLER et al. [10] have shown that nasal resistance presents substantial variability and may increase during sleep. Therefore, it is plausible to hypothesise that OSA patients presenting with normal nasal resistance at wakefulness could demonstrate increased nocturnal nasal resistance during sleep, which might predispose to oral/oro-nasal breathing and elicit apnoea/hypopnoeas. Thus, pharmacological prevention of nocturnal nasal obstruction in such OSA patients might decrease oral/oro-nasal breathing and eventually be beneficial for OSA by decreasing apnoea/hypopnoeas. Additionally, the evaluation of this pharmacological intervention during sleep could be an important step in understanding the upper airway physiology in OSA patients.

Therefore, the present study aims to investigate whether ensuring nasal airway patency during sleep by pharmacological prevention of nasal obstruction could alter the breathing route pattern and lead to a decrease in the number of apnoea/hypopnoeas in OSA patients with normal nasal resistance at wakefulness. A combination of a fast-acting nasal decongestant with a nasal corticosteroid, which can attenuate nasal inflammation associated with OSA [11, 12], will be used. The hypothesis that we aimed to test is that the application of this combination decreases oral/oro-nasal breathing and subsequently AHI.

\section{Methods}

Study subjects

Consecutive patients who referred from April 2010 to January 2012 to the Center of Sleep Disorders of "Evangelismos" General Hospital of Athens, Athens, Greece, for suspected sleep disordered breathing were recruited. The enrolment criteria were as follows. 1) AHI $>10$ events per hour at baseline; 2) normal nasal resistance measured in a supine position with active anterior rhinomanometry $\left.\left(<3.0 \mathrm{cmH}_{2} \mathrm{O} \cdot \mathrm{L}^{-1} \cdot \mathrm{s}\right) ; 3\right)$ no recent surgery involving the upper airway; 4) fewer than three central apnoeas per hour or $5 \%$ of total apnoeas; 5) no use of medications known to influence nasal resistance (antihistamine and decongestants, etc.); 6) no upper or lower respiratory tract disease, including a history of nasal allergy; and 7) no smoking. Exclusion criterion was considered the treatment of OSA with continuous positive airway pressure (CPAP) during the course of the study. Prior to enrolment in the study, each participant provided signed informed consent. The study was approved by the hospital ethics committee.

\section{Protocol}

A randomised, double-blind, placebo-controlled, crossover design was used (fig. 1). Using a table of random numbers, subjects were randomised into two groups. The patients of the first group initially underwent 1 week of therapy with nasal tramazoline and dexamethasone (application twice per day) followed by a 2 -week washout period and 1 week of therapy with an identically looking nasal placebo (sodium chloride, $0.9 \%$ solution; application twice per day). The patients of the second group initially underwent 1 week of therapy with nasal placebo followed by 2 weeks of washout period and 1 week of therapy with nasal tramazoline and dexamethasone. A 2-week washout period between the 1-week regimens was employed because the time needed for the effect of medication to disappear is $\sim 1$ week $[13,14]$. The patients underwent four assessments, at the start and end of each treatment period. Every assessment consisted of an overnight polysomnography with concomitant measurement of breathing route pattern (oral, nasal and oro-nasal breathing epochs) as previously described [9], along with nasal resistance measurement. The present study is registered on the publicly accessible database www.clinicaltrials.gov (NCT01601509). 
FIGURE 1 Study protocol. The study was undertaken according to a randomised, placebo-controlled, crossover design. After the first assessment, patients underwent a 1-week treatment with either nasal tramazoline with dexamethasone or nasal placebo, followed by a washout period of 2 weeks and a 1 -week treatment with the other regimen. At the start and end of each treatment period, patients underwent an assessment that consisted of an overnight polysomnography with concomitant measurement of breathing route pattern along with nasal resistance measurement.

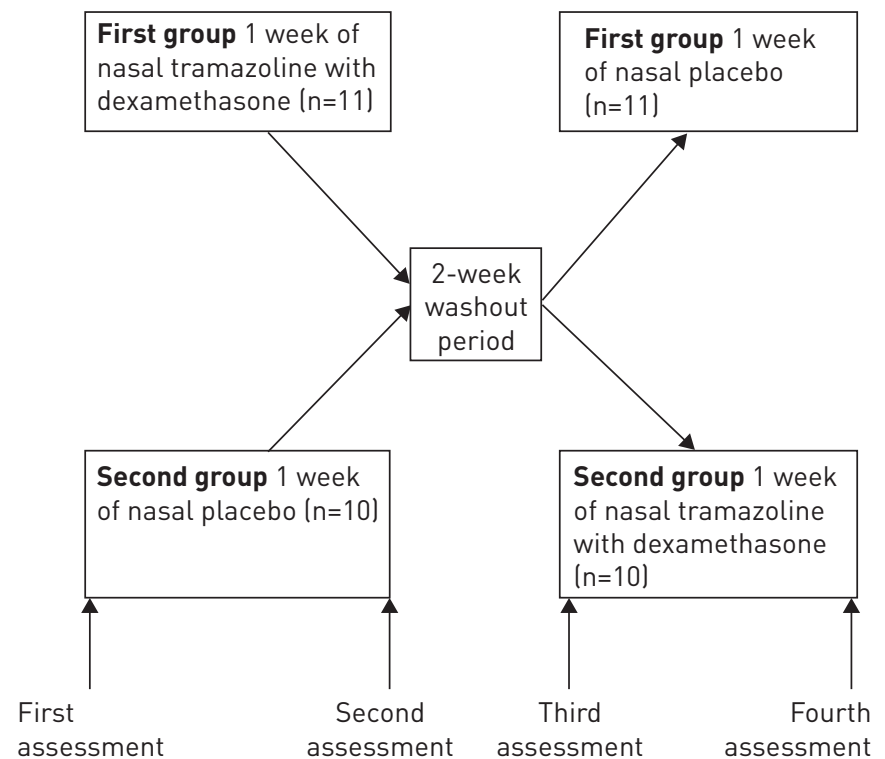

\section{Rhinomanometry}

For each subject, nasal resistance to airflow was measured on the night of the polysomnography during wakefulness without decongestion, first, in the upright seated position and then in supine position after lying down for $10 \mathrm{~min}$, by active anterior rhinomanometry (PDD-301/r, Piston, Budapest, Hungary) using a standard protocol [9]. International recommendations were followed during the procedure [15]. In brief, patients wore a closely fitting face mask that did not distort the nostrils or the nasal valve and breathed through only one nostril (first the left and then the right) with the mouth closed. The pressure probe was placed at the opening of the contralateral occluded nostril not being tested. Total resistance was then automatically calculated from the two unilateral measurements. Nasal resistance was given at a pressure difference of $150 \mathrm{~Pa}$ across the nasal passage. Anterior rhinomanometry requires minimal cooperation, and has increased reproducibility rate and minimal failure rate [15].

\section{Polysomnographic methods}

A full-night of diagnostic polysomnography (EMBLA S7000; Medcare Flaga, Reykjavik, Iceland) was performed in each subject. To determine the stages of sleep, an electroencephalogram (with four channels: C4-A1, C3-A2, O2-A1 and O1-A2), electro-oculogram and electromyogram of the submentalis muscle were obtained. Arterial blood oxyhaemoglobin was recorded with the use of a finger pulse oximeter. Thoracoabdominal excursions were measured qualitatively by respiratory effort sensors (XactTrace belts featuring Respiratory Inductive Plethysmography (RIP); Medcare Flaga) placed over the rib cage and abdomen. Snoring was detected with a vibration snore sensor and body posture with a body position sensor. Airflow was monitored using an oral thermistor (oral flow sensor; Medcare Flaga) placed in front of the mouth and a nasal cannula/pressure transducer $(21 \mathrm{inch} / 53 \mathrm{~cm}$; Medcare Flaga) inserted into the opening of the nostrils and linked to independent channels, as previously described [9]. All variables were recorded with a digital acquisition system (Somnologica 3.3; Medcare Flaga).

\section{Pharmacological therapy}

A combination of a nasal decongestant (tramazoline hydrochloride $120 \mu \mathrm{g}$ ) with a nasal corticosteroid (dexamethasone-21 isonicotinate $20 \mu \mathrm{g}$ per dose) was used (Dexa-Rhinaspray N; Boehringer Ingelheim, Ingelheim, Germany). Tramazoline (an imidazoline derivative) is a nasal decongestant that attains its maximal effect at between 30 and $210 \mathrm{~min}$ after application [11], while nasal dexamethasone can attenuate nasal inflammation associated with OSA [12].

\section{Data analysis}

The code of the medication was maintained during randomisation and was broken only after the completion of data analysis. Sleep stage was scored manually in 30-s epochs [16]. Obstructive respiratory events were scored using standard criteria $[16,17]$. Thus, apnoea was defined as the absence of airflow for $>10 \mathrm{~s}$ in the presence of continued respiratory efforts [16]. Hypopnoea was defined as the reduction in chest wall 
movement to an amplitude that was smaller than $\sim 70 \%$ of the baseline level, lasting $>10 \mathrm{~s}$, and leading to a decrease in haemoglobin saturation of at least $4 \%$ [17]. The number of episodes of apnoeas and hypopnoeas per hour of sleep is referred to as the AHI, whereas the number of episodes of apnoeas, hypopnoeas and respiratory effort-related arousals per hour of sleep is referred to as the respiratory distress index [16].

The route of breathing was evaluated by using the oral and nasal sensor signals to classify each 30 -s epoch as nasal, oral or oro-nasal based on the predominant breathing route, and was expressed in \% total sleep epochs (TSE), as previously described [9]. Cross-contamination between the oral and nasal channel was meticulously excluded by regular testing during polysomnographic calibration. Thus, we asked subjects to breathe normally and exclusively through the nose for $30 \mathrm{~s}$ and, subsequently, through the mouth for another $30 \mathrm{~s}$ in both supine and lateral postures so that we could verify that each sensor was activated exclusively. We continuously checked sensors during the recording to avoid dislocation. All measurements were analysed by a single investigator to ensure consistency and all polysomnographies were scored by a single experienced sleep technologist and subsequently reviewed by the same investigator, who was blinded to the patient's group identity.

\section{End-points}

The primary outcome was AHI. Secondary outcomes included nasal resistance values, breathing route pattern, and the Epworth Sleepiness Scale (ESS) score as a measure of subjective sleepiness [18].

\section{Statistical analysis}

The minimum sample size was calculated on the basis of $80 \%$ power and a two-sided 0.05 significance level ( $\mathrm{G}^{\star}$ Power software version 3.0.10; Franz Faul, Kiel University, Kiel, Germany). A sample size capable of detecting a change of 5 events per hour for AHI after pharmacological intervention was estimated using the standard deviations obtained from our previous study [8]. The critical sample size was estimated to be 19 patients. Values are presented as mean $\pm \mathrm{SD}$ or median (interquartile range) after testing for normal distribution using the Kolmogorov-Smirnov test. Depending on the distribution of variables, either parametric (paired t-test) or nonparametric (Wilcoxon signed rank and Spearman's rank) tests were used. Data were analysed according to the method of JONES and KENWERD [19]. Comparison of data at the entry to each study period, i.e. at the start of the study and at the end of the washout period, was performed using the paired t-test. The treatment effect for each variable was estimated using the difference between the value at the end of treatment minus the value at the beginning of treatment. Treatment effect differences between nasal tramazoline with dexamethasone and nasal placebo were compared using the Wilcoxon signed rank test. Relationships between variables were determined by the Spearman's rank correlation coefficient $\left(R_{s}\right)$. A two-tailed p-value of $<0.05$ was considered statistically significant.

\section{Results}

In total, 21 patients (13 males) were enrolled and completed the study uneventfully. The baseline demographics and patients characteristics are detailed in table 1.11 patients were randomly assigned to the first group and 10 patients to the second group (fig. 1).

\section{TABLE 1 Anthropometric data and baseline sleep parameters and nasal resistance values}

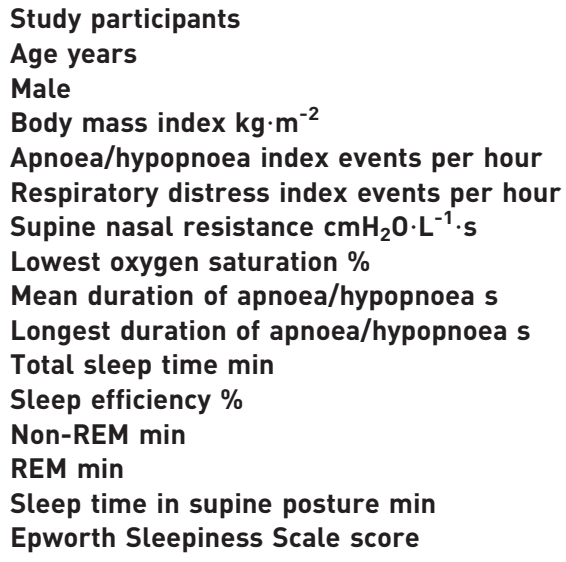

21

$38.0+7.7$

$13(62)$

$30.7 \pm 2.9$

$31.1+14.8$

$33.6 \pm 17.5$

$2.2+0.3$

$84.7 \pm 4.2$

$18.8 \pm 5.8$

$27.5 \pm 7.9$

$304.5 \pm 68.3$

$88.4 \pm 13.3$

$248.9 \pm 57.8$

$55.6+21.8$

$155.5 \pm 104.0$

$9.9 \pm 3.6$

Data are presented as $n, n(\%)$ or mean \pm SD. REM: rapid eye movement. 
TABLE 2 Patient characteristics at the entry to each study period

Start of study

End of washout period"

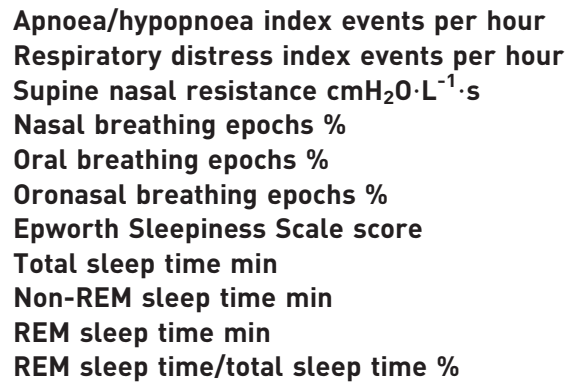

$$
\begin{gathered}
31.1 \pm 14.8 \\
33.6 \pm 17.5 \\
2.2 \pm 0.3 \\
64.3 \pm 6.8 \\
2.4 \pm 1.4 \\
33.3 \pm 4.6 \\
9.9 \pm 3.6 \\
304.5 \pm 68.3 \\
248.9 \pm 57.8 \\
55.6 \pm 21.8 \\
18.3 \pm 3.8 \\
88.4 \pm 13.3
\end{gathered}
$$

$28.8 \pm 11.9$

$31.2+19.2$

$2.1 \pm 0.4$

$67.0 \pm 6.2$

$2.1 \pm 1.5$

$30.9 \pm 4.9$

$8.8 \pm 2.0 *$

$345.8 \pm 43.7^{*}$

$242.6 \pm 49.9$

$103.2 \pm 42.5^{*}$

$29.8 \pm 4.3^{*}$

$92.5 \pm 10.1$

Data are presented as mean \pm SD. REM: rapid eye movement. \#: first assessment in figure 1; ${ }^{\text {I: third }}$ assessment in figure 1. *: $\mathrm{p}<0.05$ versus start of study (paired t-test).

\section{Study entry and post washout}

Patient characteristics at the entry to each period of the study are shown in table 2. No carryover effect on the measured parameters was observed. As can be seen, there was no significant difference in AHI, respiratory distress index, nasal resistance and breathing route pattern between the entry to the first period of the study (fig. 1; first assessment) and the end of the washout period (fig. 1, third assessment), i.e. entry to the second period of the study. Only total and REM sleep time increased in the third assessment compared with the first assessment.

\section{Treatment effect differences}

Treatment effect differences are summarised in table 3. AHI, AHI in supine position, respiratory distress index, nasal resistance, oral breathing epochs, oro-nasal breathing epochs and non-REM sleep time decreased, whereas nasal breathing epochs, minimum oxygen saturation and REM sleep time increased with nasal tramazoline and dexamethasone compared with nasal placebo. ESS score change was not different between the nasal tramazoline and dexamethasone and nasal placebo groups.

The change of AHI after 1 week of therapy with nasal tramazoline with dexamethasone was inversely related to the change of nasal breathing epochs $\left(R_{s}=0.78 ; p<0.001\right)$ (fig. 2a). Conversely, the change of AHI after 1 week of therapy with nasal placebo did not correlate with the change of nasal breathing epochs $\left(R_{s}=0.38\right.$; $\mathrm{p}=0.055$ ) (fig. $2 \mathrm{~b}$ ). Additionally, the change of nasal resistance after 1 week of therapy with nasal tramazoline with dexamethasone did not correlate with either the change of AHI $\left(\mathrm{R}_{\mathrm{s}}=0.126 ; \mathrm{p}=0.234\right)$ or the change of nasal breathing epochs $\left(\mathrm{R}_{\mathrm{s}}=0.228 ; \mathrm{p}=0.095\right)$.

\section{Effect of treatment with nasal tramazoline and dexamethasone on AHI}

Individual and mean values of AHI at the start and end of the treatment period with nasal tramazoline and dexamethasone are shown in figure 3. There was a mean decrease of AHI by $21 \%$. Among 21 patients, three (14\%) had post-treatment AHI $<10$ events per hour.

\section{Discussion}

The main findings of this randomised, placebo-controlled, double-blind, crossover trial on the effects of a 1week application of nasal tramazoline with dexamethasone in OSA patients with normal nasal resistance at wakefulness were that this therapy: 1) is associated with a decrease in oro-nasal breathing epochs and a concomitant increase in nasal breathing epochs; 2) is associated with a mean decrease in AHI by $21 \%$; and 3 ) the change in nasal breathing epochs is inversely related to the change in AHI, so that the increase in nasal breathing epochs explains $63.6 \%$ of the variance of the decrease in AHI.

The findings of this study corroborate the pathophysiological pathway between nasal breathing and OSA. Indeed, given that nasal resistance measured at the beginning of the night is lower by $26 \pm 20 \%$ than subsequent measurements, it was assumed that nocturnal increased nasal resistance might play a role in the appearance of apnoeas in OSA patients with normal nasal resistance at wakefulness [10]. The current study 
TABLE 3 Treatment effect differences

Nasal tramazoline with

dexamethasone

Apnoea/hypopnoea index events per hour

Respiratory distress index events per hour

Supine nasal resistance $\mathrm{cmH}_{2} \mathrm{O} \cdot \mathrm{L}^{-1} \cdot \mathrm{s}$

Nasal breathing epochs \% TSE

Oral breathing epochs \% TSE

Oronasal breathing epochs \% TSE

Minimum oxygen saturation \%

Average oxygen saturation \%

Epworth Sleepiness Scale score

Total sleep time min

Non-REM sleep time min

REM sleep time min

Sleep efficiency \%

Sleep time in supine position $\%$

Apnoea/hypopnoea index in supine position events per hour

$$
\begin{gathered}
-6.1(-10.7--2.2) \\
-8.2(-12.8--4.1) \\
-0.6(-0.8--0.3) \\
13.0(9.5-16.6) \\
-0.8(-1.9--0.4) \\
-10.0(-13.2--7.6) \\
0.6(-0.2-0.9) \\
0.9(-0.1-1.9) \\
-1.0(-4.0-0.0) \\
4.6(-7.2-15.9) \\
-16.6(-36.2--7.9) \\
23.6(5.2-35.9) \\
2.9(0.8-10.2) \\
1.0(-1.2-4.2) \\
-3.1(-8.9-7.6)
\end{gathered}
$$

Data are presented as median (interquartile range). TSE: total sleep epochs; REM: rapid eye movement. ${ }^{* *}$ : $<<0.01$ versus nasal tramazoline with dexamethasone (Wilcoxon signed rank test).

adds to the literature by recruiting OSA patients with normal nasal resistance at wakefulness and demonstrating that the pharmacological prevention of the potential nocturnal increase of nasal resistance changes the breathing route pattern of OSA patients and decreases AHI, suggesting a strong correlation between restoration of nasal breathing and improvement of AHI. Indeed, all previous studies examining the role of nasal airway in sleep-disordered breathing have recruited OSA patients with nasal pathology or increased nasal resistance at baseline [6-8].

The factors that could explain the aforementioned correlation have been thoroughly studied in several trials, and are associated either with mouth opening/breathing or absence of nasal breathing. Thus, Meurice et al. [20] demonstrated that mouth opening increases upper airway collapsibility due to a combination of upper airway narrowing and a decrease in the efficiency of upper airway dilator muscle contraction. Further evidence in the same direction was added by MORIKAWA et al. [21] who observed that opening the jaw decreased the mean distance between the tongue and posterior pharyngeal wall from 17.5
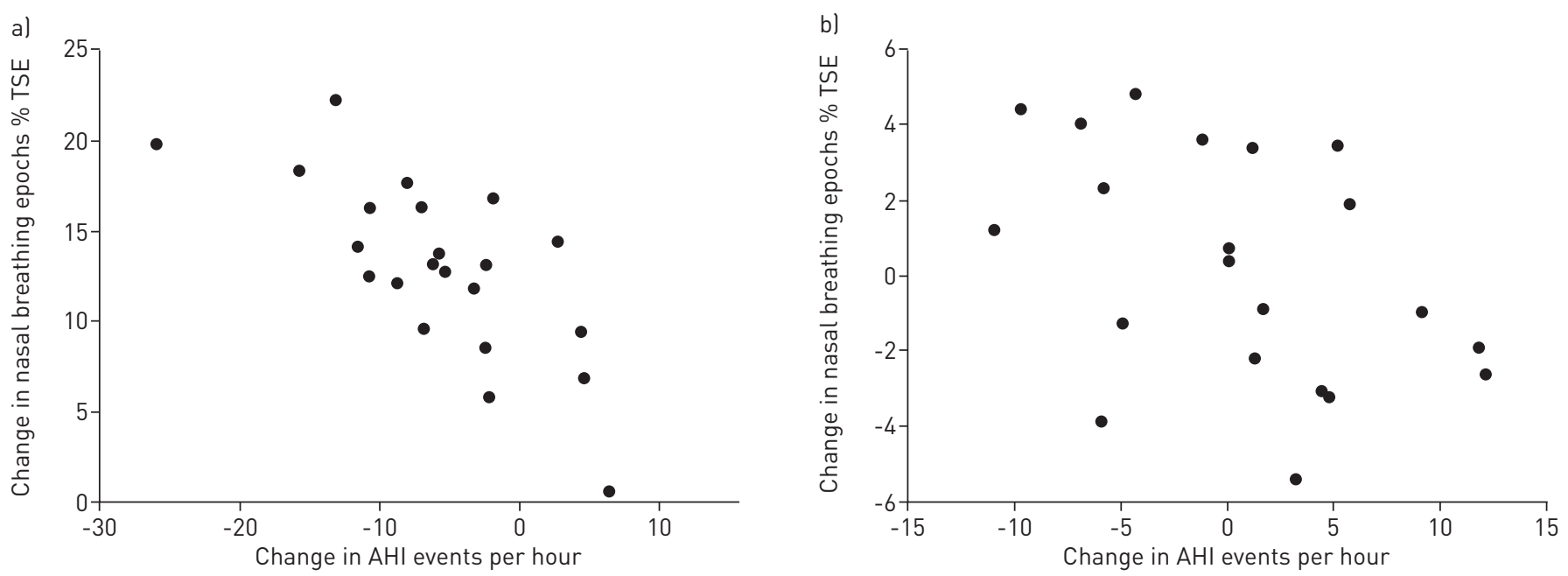

FIGURE 2 Relationship between the change in nasal breathing epochs and the change in apnoea/hypopnoea index (AHI) a) after 1 week of therapy with nasal tramazoline with dexamethasone $\left(R_{s}=0.78 ; p<0.001\right)$ and $\left.b\right)$ after 1 week of therapy with nasal placebo $\left(R_{s}=0.38 ; p=0.055\right)$. TSE: total sleep epochs. 
FIGURE 3 Individual (circles) and mean (horizontal lines) values of apnoea/hypopnoea index (AHI) at the start and end of treatment period with nasal tramazoline and dexamethasone.

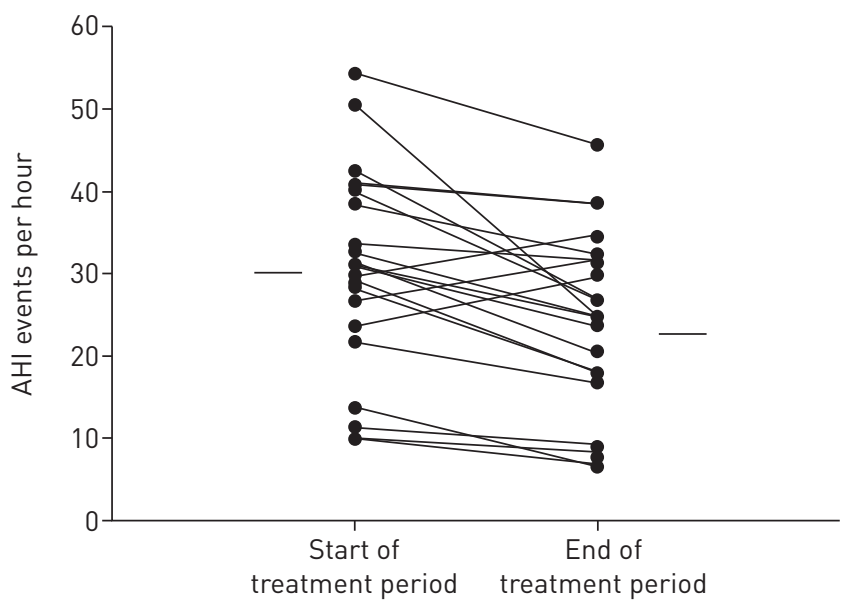

to $11 \mathrm{~mm}$. Similarly, KUNA and REMMERS [22] suggested that mouth opening is associated with an inferior movement of the mandible, compromising pharyngeal diameter. Combining the above observations with cephalometric studies [23], which demonstrate that apnoeics have a mandible that is more displaced to the posterior than normal subjects, it is not surprising that, in OSA patients, mouth opening leads easier to upper airway collapse. Additionally, there are data suggesting that irritation of nasal airflow-sensitive receptors during nasal breathing is important in maintaining upper airway patency by increasing oropharyngeal muscle activity. In fact, WHITE et al. [24] blocked these receptors using 4\% lidocaine local anaesthesia and provoked a four-fold increase in the sleep-disordered breathing events, and BASNER et al. [25] measured increased genioglossal and alae nasi electromyographic activity in awake humans breathing through the nose.

There are few randomised studies that all, as opposed to our study, recruited OSA patients with increased nasal resistance at baseline and investigated the effect of nasal medication on nocturnal breathing disturbances. In fact, KERR et al. [26] studied the effect of oxymetazalone in combination with a vestibular stent and found no change in AHI or in sleep efficiency, and an only minor reduction in arousal index despite a considerable reduction in nasal resistance. Furthermore, MCLEAN et al. [27] applied oxymetazoline twice during the night in combination with an external nasal-valve dilator strip. This intervention was associated with a modest decrease in AHI and an improved sleep efficiency and architecture. KIELY et al. [7] investigated the effect of nasal corticosteroid in OSA patients with rhinitis and found a modest reduction of nasal resistance, which was positively correlated with a decrease in AHI. Lastly, Clarenbach et al. [13] applied once a nasal decongestant and measured nasal conductance continuously during sleep. The authors found reduced AHI during maximal nasal decongestion and thus suggested a pathophysiological link between nasal resistance and sleep disordered breathing. The findings of most of the aforementioned trials are in accordance with the results of the current study, which also shows a modest decrease in AHI and an improvement in sleep architecture, as indicated by the increase in rapid eye movement sleep time (table 3 ).

Besides the importance of the findings of the current study in elucidating upper airway physiology in OSA patients with normal nasal resistance at baseline, in terms of clinical relevance, AHI decreased by $21 \%$ and only three out of 21 patients, notably those with the lowest AHI at baseline, had post-treatment AHI $<10$ events per hour. Therefore, our data suggest that the use of the combination of a nasal decongestant with a corticosteroid is not an effective treatment for OSA patients and could only be regarded as potential therapy for the less severe cases (i.e. AHI $<15$ events per hour) or as a complementary treatment to other treatment modalities. Moreover, the possibility of side-effects from the long-term use of the combination of nasal decongestant with nasal steroid should be borne in mind [28]. Indeed, each course of treatment should not exceed 14 days, because prolonged use has been associated with rebound congestion of the nasal mucosa and systemic effects of corticosteroids [28]. Consequently, it is evident that the benefits obtained by the combination of medication used in the current trial could only be achieved safely if used on a shortterm basis and, thus, the clinical relevance of our findings in the long-term remains unknown.

Some issues and possible weaknesses of the current study must be acknowledged and deserve consideration. First, the study was adequately powered to detect an improvement in AHI of 5 events per hour, because any less improvement is unlikely to be clinically relevant. Secondly, the instrumentation of nasal cannula/ 
pressure transducer and oral thermistor to detect airflow presents some drawbacks that have been thoroughly discussed previously [9]. Although these devices are nonobtrusive and easily tolerated, their signal-flow relationship is nonlinear, resulting in underestimation of nasal ventilation and overestimation of oral ventilation, especially at low flows [9]. Therefore, it could be possible that oral-only breathing may still have a nasal component, and any detection of oral only breathing might actually be scarce. Thirdly, although sensor dislocation from the nose or mouth was meticulously checked by the technician on duty, it is possible that slight deviations in thermistor position may not have been completely avoided, and this may have then resulted in nasal airflow contamination of the oral signal. Fourthly, in the current study, nasal resistance was not measured continuously and, thus, it was not possible to directly, minute by minute, evaluate the association between nocturnal nasal resistance with the change in nasal breathing epochs and AHI. Fifthly, the increase in rapid eye movement and total sleep time at the third assessment (table 2) could be attributed to the so called "first-night effect" [29]. Finally, ESS is not designed to assess changes in the propensity to fall asleep within 1 week. However, experience with the rapid changes in subjective sleepiness that occur when OSA patients are treated with CPAP suggests that ESS might be an adequate measure of fluctuations in sleepiness, even on a short-term basis [30].

In conclusion, the findings of the present study suggest that a 1-week application of nasal tramazoline with dexamethasone in OSA patients with normal nasal resistance at wakefulness decreases AHI by increasing nasal breathing epochs. However, the decrease in AHI is modest and, thus, the therapeutic role of preserving nasal airway patency on OSA severity remains rather limited.

\section{References}

Proctor DF. Nasal physiology and defense of the lungs. Am Rev Respir Dis 1977; 115: 97-129.

Fitzpatrick M, Driver H, Chata N, et al. Partitioning of inhaled ventilation during sleep in normal subjects. J Appl Physiol 2003; 94: 883-890.

Gleeson K, Zwillich C, Braier K, et al. Breathing route during sleep. Am Rev Respir Dis 1986; 134: 115-120.

4 Lieberman A, Okhi M, Forte V, et al. Nose/mouth distribution of respiratory airflow in 'mouth breathing children'. Acta Otolaryngol (Stockh) 1990; 109: 454-460.

5 Lofaso F, Coste A, D’Ortho M, et al. Nasal obstruction as a risk factor for sleep apnoea syndrome. Eur Respir J 2000; 16: 639-643.

6 Kohler M, Bloch KE, Stradling JR. The role of the nose in the pathogenesis of obstructive sleep apnoea and snoring. Eur Respir J 2007; 30: 1208-1215.

7 Kiely JL, Nolan P, McNicholas WT. Intranasal corticosteroid therapy for obstructive sleep apnoea in patients with co-existing rhinitis. Thorax 2004; 59: 50-55.

8 Koutsourelakis I, Georgoulopoulos G, Perraki E, et al. Randomised trial of nasal surgery for fixed nasal obstruction in obstructive sleep apnoea. Eur Respir J 2008; 31: 110-117.

9 Koutsourelakis I, Vagiakis E, Roussos C, et al. Obstructive sleep apnoea and oral breathing in patients free of nasal obstruction. Eur Respir J 2006; 28: 1222-1228.

10 Kohler M, Thurnheer R, Bloch KE. Side-selective, unobtrusive monitoring of nasal airflow and conductance. J Appl Physiol 2006; 101: 1760-1765.

11 Hochban W, Althoff $\mathrm{H}$, Ziegler A. Nasal decongestion with imidazoline derivatives: acoustic rhinometry measurements. Eur J Clin Pharmacol 1999; 55: 7-12.

12 Rubinstein I. Nasal inflammation in patients with obstructive sleep apnea. Laryngoscope 1995; 105: 175-177.

13 Clarenbach CF, Kohler M, Senn O, et al. Does nasal decongestion improve obstructive sleep apnea? J Sleep Res 2008; 17: 444-449.

14 Wilson AM, Sims EJ, McFarlane LC, et al. Effects of intranasal corticosteroids on adrenal, bone, and blood markers of systemic activity in allergic rhinitis. J Allergy Clin Immunol 1998; 102: 598-604.

15 Clement PAR, Gordts F. Consensus report on acoustic rhinometry and rhinomanometry. Rhinology 2005; 43: 169-179.

16 American Academy of Sleep Medicine Task Force. Sleep-related breathing disorders in adults: recommendations for syndrome definition and measurement techniques in clinical research. Sleep 1999; 22: 667-689.

Meoli A, Casey K, Clark R, et al. Hypopnea in sleep-disordered breathing in adults. Sleep 2001; 24: 469-470.

Johns M. A new method for measuring sleepiness: the Epworth Sleepiness Scale. Sleep 1991; 12: 540-545.

Jones B, Kenward MG, eds. Design and Analysis of Cross-over Trials. New York, Chapman and Hall, 1989.

20 Meurice JC, Marc I, Carrier G, et al. Effects of mouth opening on upper airway collapsibility in normal sleeping subjects. Am J Respir Crit Care Med 1996; 153: 255-259.

21 Morikawa S, Safer P, Decarlo J. Influence of the headjaw position upon upper airway patency. Anesthesiology 1961; 22: $265-270$.

22 Kuna ST, Remmers JE. Neural and anatomic factors related to upper airway occlusion during sleep. Med Clin North Am 1985; 69: 1221-1242.

23 Rivlin J, Hoffstein J, Kalbfleisch J, et al. Upper airway morphology in patients with idiopathic obstructive sleep apnea. Am Rev Respir Dis 1984; 129: 355-360.

24 White D, Cadieux R, Lombard R, et al. The effects of nasal anesthesia on breathing during sleep. Am Rev Respir Dis 1985; 132: 972-975.

25 Basner R, Simon P, Schwartzstein R, et al. Breathing route influences upper airway muscle activity in awake normal adults. J Appl Physiol 1989; 66: 1766-1771.

26 Kerr P, Millar T, Buckle P, et al. The importance of nasal resistance in obstructive sleep apnea syndrome. J Otolaryngol 1992; 21: 189-195. 
27 McLean HA, Urton AM, Driver HS, et al. Effect of treating severe nasal obstruction on the severity of obstructive sleep apnoea. Eur Respir J 2005; 25: 521-527.

28 Milgrom H, Bender B. Adverse effects of medications for rhinitis. Ann Allergy Asthma Immunol 1997; 78: 439-444.

29 Scholle S, Scholle HC, Kemper A, et al. First night effect in children and adolescents undergoing polysomnography for sleep-disordered breathing. Clin Neurophysiol 2003; 114: 2138-2145.

30 Hardinge FM, Pitson DJ, Stradling JR. Use of the Epworth Sleepiness Scale to demonstrate response to treatment with nasal continuous positive airways pressure in patients with obstructive sleep apnea. Respir Med 1995; 89: $617-620$. 